\title{
Special issue on soft computing in economic application
}

\author{
Hung T. Nguyen ${ }^{1,2} \cdot$ Vladik Kreinovich $^{3}$ \\ Accepted: 5 April 2021 / Published online: 16 April 2021 \\ (c) The Author(s), under exclusive licence to Springer-Verlag GmbH Germany, part of Springer Nature 2021
}

Engineering and science are very important for our lives: science provides fundamental ideas that engineers transform into useful designs, from a computer on which we are preparing this editorial to cars on which we drive to our offices to refrigerators that store our lunches. However, the ultimate reason why new inventions appear and why gadgets make our lives better year after year is economy. Without proper economic incentives, even the best inventions would not translate into practical applications. Suffice it to say that, according to historians of science, steam engines were invented in ancient Alexandria two thousand years ago—-but they were only used to spectacularly open the doors of a temple on big celebrations. Some popular books about the Mayan culture claim that the Mayans did not know the wheel-since they did not use wheels for transportation. This is a misunderstanding; Mayans were smart enough to invent a wheel many thousand years ago, they have numerous children's toys with perfect wheels-but just like the ancient Greeks, and they did not have the economic incentive to actively use their inventions.

From this viewpoint, economics-the study of economic phenomena-is as important as science and engineering, if not more important. But there is a problem. In engineering, we mostly know the equations that describe the desired phenomena: we know the equations that describe the trajectory of a spaceship, we know the equations that describe how electrons move inside a computer, and we know how refrigerators work. The corresponding problems may be complex, but they are mostly well defined.

Economics is different. There are some equations describing economic phenomena-although we need to be cautious and take into account that our equations and the underlying models are only a crude approximation; otherwise, we may get a repetition of the 2008 crisis (which was largely brought

Vladik Kreinovich

vladik@utep.edu

1 New Mexico State University, Las Cruces, New Mexico, USA

2 Chiang Mai University, Chiang Mai, Thailand

3 University of Texas at El Paso, Texas, El Paso, USA in by exactly such lack of caution). However, for a large number of economic phenomena, no equations are known.

Because of this difference, while a successful engineer uses equations and comes up with a great design, a CEO of a successful company or bank is not successful because he or she uses equations-in most cases, the CEO is successful because he/she follows good imprecise rules and ideas. Many of these successful CEOs are willing to share the secrets of their success-every bookstore has a whole section of books written by successful entrepreneurs. Do these books contain any equations or at least technical details-as do similar books written by successful scientists? No, the advice of these entrepreneurs is informal, imprecise, and thus, not easy to follow for those who do not have their intuition, their understanding. Let us given an example. If someone teaches us the laws of perspective, we can draw realistic pictures of buildings, but if the only artist's advice is to make it beautiful and realistic, this will not help us much.

To make economic advice useful, we need to translate it into more precise language. This, in a nutshell, is one of the main ideas behind soft computing - that we translate imprecise rules into precise control strategies (fuzzy logic), that we make computes emulate how we learn from examples (neural networks), that we simulate natural selection to come up with better solutions (evolutionary algorithms), etc. The use of soft computing techniques in economic applications is the main topic of this special issue.

Of course, we are still far away from solving all the problems facing economics, but each of the numerous papers from this special issue is an important step towards solving these problems. We expect that those readers who are interested in economic applications will realize, after reading these papers, that soft computing methods have a great potential and that-in contrast to engineering where soft computing methods are actively and effectively used-soft computing methods are drastically underused in economics. On the other hand, those readers who are developing new soft computing techniques and applying them in engineering will hopefully better understand how such methods can be used in 
economics as well—and all together, we will progress even further.

In short, we believe that this issue will be successful-in the sense that it will lead to new applications, new results, and new developments. And when this indeed happens, this success will be due to many people who, directly and indirectly, contributed to this special issue. First, the authors: without their very interesting papers, there would have been no issue. Even authors of rejected papers contributed-we were very selective, but, based on what referees described, even most rejected papers had many interesting and promising ideas, ideas which may not be ready for publication right now, but that will eventually lead to excellent future papers. Of course, the referees need to be thanked-in this issue, where papers need to be understood both by economists and by specialists in soft computing, it was not easy to find referees who have a good understanding of both, so the referees who agreed to serve had to review more papers that usual. Also, we need to thank the editors of the journal, who believed in our vision, who tolerated our — understandable but still—delays. And last but not the least, we want to thank all the readers of this issue, all those who do not hesitate to spend additional efforts in reading and understanding these not-very-easy-toread interdisciplinary papers. Many thanks to you all, and we hope to see many such issues in the future!

Hung T. Nguyen and Vladik Kreinovich, April 2021

Publisher's Note Springer Nature remains neutral with regard to jurisdictional claims in published maps and institutional affiliations. 